\title{
Cassava/sugar palm fiber reinforced cassava starch hybrid composites: physical, thermal and structural properties
}

\begin{abstract}
A hybrid composite was prepared from cassava bagasse (CB) and sugar palm fiber (SPF) using casting technique with cassava starch (CS) as matrix and fructose as a plasticizer. Different loadings of SPF (2, 4, 6 and $8 \%$ w/w of dry starch) were added to the CS/CB composite film containing $6 \% \mathrm{CB}$. The addition of SPF significantly influenced the physical properties. It increased the thickness while decreasing the density, water content, water solubility and water absorption. However, no significant effect was noticed on the thermal properties of the hybrid composite film. The incorporation of SPF increased the relative crystallinity up to $47 \%$, compared to $32 \%$ of the CS film. SEM micrographs indicated that the filler was incorporated in the matrix. The film with a higher concentration of SPF (CS$\mathrm{CB} / \mathrm{SPF} 8$ ) showed a more heterogeneous surface. It could be concluded that the incorporation of SPF led to changes in cassava starch film properties, potentially affecting the film performances.
\end{abstract}

Keyword: Cassava film; Hybrid composite; Cassava bagasse; Sugar palm fiber; Physical properties; Thermal properties 